\title{
Pensamiento consciente, ignorancia científica y el avance de la ciencia política
}

\author{
FABRicio H. Chagas Bastos*
}

La ciencia política se ha inspirado desde hace mucho en la economía como el modelo de ciencia que se debe perseguir. Asimismo, la apelación de los métodos en ciencia política es similar a la que ha pasado con la economía a partir de la década de 1970: una mezcla entre fetichismo e infalibilidad. No es sorprendente que el culto, confusión y asociación entre métodos y técnicas cuantitativas en ciencia política hayan creado un precipicio entre lo que es el logos de una investigación y las técnicas usadas para abordarlo.

Giovanni Sartori por décadas ha traído a discusión un particular problema común entre politólogos — trabajando en cualquier campo, política comparada, relaciones internacionales (RRII), política nacional—: la falta de conciencia sobre lo que están haciendo con respecto a la selección de preguntas, métodos, técnicas apropiadas y, al final, el marco conceptual y teórico utilizado para llevar a cabo la investigación. Lo que hace a Sartori un clásico es que la falta de conciencia académica de generaciones de politólogos parece haber ido creciendo y ha llevado a la disciplina hacia la irrelevancia.

Con la academia bajo la presión por publicar más y más al costo de perecer (en la inserción laboral, comités de promoción de carrera, búsqueda por financiación, etc.), existe un privilegio por las técnicas (cuantitativa o cualitativa) en vez de por el pensar en los pasos lógicos detrás de un cuestionamiento investigativo para lograr un resultado, lo que hace a este problema cada día peor.

\footnotetext{
* University of Cambridge / The University of Melbourne. Correo electrónico: fhc25@, cam.ac.uk ORCID: orcid.org/0000-0002-3533-9034
} 
En este ensayo, discuto sobre la actualidad e importancia de los argumentos de Sartori para alcanzar una ciencia política más consciente de sí misma, es decir, conectada a las diferentes realidades del mundo, sin un origen pasteurizado y pretensioso de conocimiento. Retomo parte de su crítica y la posiciono en un debate actual dentro de la disciplina. Debo advertir al lector de antemano que como alguien entrenado en RRII, todos mis ejemplos y argumentos provienen de este campo de estudio. Esta es mi contribución para honrar la memoria de Giovanni Sartori.

El presente ensayo se encuentra dividido en dos partes. En la primera sección hago una breve revisión del argumento de Sartori sobre la malformación de conceptos y lo que es un pensador consciente. Seguido a esto, continuaré con la crítica sobre el pensamiento no consciente en ciencia política y los problemas que trae para la disciplina, ejemplificando que es la malformación de conceptos, como pensador consciente, lo que puede corregirla y mostrar avances cuando esto ocurre.

\section{Malformación de conceptos y el pensador inconsciente}

Sartori (1970) identifica dos problemas principales para aquellos que estudian ciencia política, la falta de una ciencia política pensada conscientemente - una crítica recurrente en su trabajo a lo largo de los años, especialmente en contra de la academia estadounidensey el estiramiento conceptual. Este último, también conocido como distensión conceptual, implica la vaga y amorfa conceptualización producida por la tendencia de "cubrir más con términos ligeros solamente diciendo menos, y decir menos de una manera mucho menos precisa" (p. 1035). La falta de una ciencia política que piensa conscientemente ocurre cuando "el pensador inconsciente no se pregunta a sí mismo por qué esta comparando, y esta negligencia explica por qué tanto trabajo comparativo provee una gran extensión de conocimiento, pero difícilmente una estrategia para adquirir y validar nuevo conocimiento" (p. 1036). 
El primero es un problema metodológico cuyo resultado es que "nuestra ganancia en extensión de cobertura tiende a estar igualada con la pérdida de precisión connotativa" (p. 1035). Sartori (1970) propone como solución lo que él llama escalera de abstracción, es decir, la escala de abstracción de un concepto partiendo de categorías de nivel bajo — basadas en conceptualizaciones configurativas y análisis entre países, que lleva a la definición del concepto de manera contextual con una gran cohesión interna y una mínima extensión-, pasando por las categorías de nivel medio - las cuales se caracterizan por la conceptualización general, que conforma una teoría de rango medio, con conceptos definidos por el análisis-, llegando a las categorías de nivel alto — donde la conceptualización universal se da a través de la comparación de múltiples campos sobre contextos heterogéneos, junto con conceptos formados a partir de la negación, con una cohesión interna mínima y una gran extensión- (pp. 1043-1045). A partir de la escala de Sartori, los investigadores están aptos para someterse primero a una serie de preguntas, antes de aplicar directamente por la fuerza técnicas a su investigación.

El segundo problema que encontramos es la ignorancia científica. Llamo al pensador inconsciente ignorante de manera intercambiable. No hay intención de ofender con esta expresión, más bien se trata de simplificar el concepto de conciencia: la habilidad de una persona para pensar su propio campo de estudio (en este caso la ciencia política o las RRII) siguiendo un proceso lógico detrás de un cuestionamiento investigativo para encontrar ciertos resultados, en lugar de simplemente aplicar una técnica a un problema que busca la generación de un resultado. Aquellos que no tienen tales habilidades, dada la definición de Sartori, son científicos ignorantes con respecto a cómo se debe pensar el mundo intelectual en el cual se encuentran inmersos.

Ambos problemas se encuentran umbilicalmente conectados dado que cuando se aplica una técnica a un problema, en vez de pasos lógicos detrás de un cuestionamiento investigativo, y se estiran conceptos para que encajen con ciertos datos buscando generar un resultado - en general, un artículo para un diario revisado por pares-, las comuni- 
dades de la ciencia política y las RRII están olvidando lentamente qué es ser un pensador consciente.

\section{Ignorancia y conciencia}

La inconformidad de Sartori con respecto a cómo estaba evolucionando la ciencia política comenzó a principios de 1970 —en Concept Misformation in Comparative Politics - y poco cambió hasta 2004, cuando publicó un pequeño comentario preguntando provocativamente ¿Hacia dónde va la ciencia política? — "W here is Political Science Going?'. Su conclusión va directo al punto: dada la simplificación excesiva del pensamiento lógico, el desarrollo de la ciencia política no va a ninguna parte.

Lo "lúgubre" (Sartori, 2004, p. 785) no es adjetivo exclusivo de la ciencia política, sino que también se aplica a sus subcampos, como las RRII, respetando sus particularidades disciplinarias. Las RRII en las últimas décadas al menos han intentado reorganizarse y readaptarse a los contextos locales y formas familiares de teorizar las relaciones internacionales, y han intentado incluir dentro del debate mainstream formas autóctonas de análisis para ayudar a solucionar problemas locales (Lima, 2011). En la disciplina, esto ha sido denominado el giro al surglobal ${ }^{1}$. En pocas palabras, el giro hacia el sur ocurre cuando las limitaciones de la teoría de RRII se encuentran con el esfuerzo de académicos alrededor del mundo por hacer una aproximación a la disciplina más plural y global. El debate producido por el centro anglosajón de la disciplina no es conveniente para explicar el mundo ni como referencia para la formulación de políticas. Leslie Armijo (2015) ilustra lo anterior cuando afirma que mientras:

en la década del 2000 los académicos estadounidenses debatían si el "momento unipolar" post Guerra Fría había o no terminado, y si podría considerarse a China como un serio competidor global.

1 Entendiendo como Sur Global a la periferia o países no occidentales. 
En Brasil y Argentina, por otro lado, casi todas las presentaciones académicas empezaban por: 'ahora que el mundo es multipolar'.

Mientras la comunidad de las RRII alrededor del mundo, y especialmente fuera del centro anglosajón de la disciplina, promueve esfuerzos para ampliar el alcance de la disciplina, en algunas partes del mundo pensadores inconscientes todavía ignoran la lógica pura y simple y retienen una mentalidad colonial. En vez de entender los avances en las definiciones y clasificaciones, enfoques teóricos, evidencia empírica proveniente de datos cuantitativos y cualitativos, algunos académicos prefieren negar la marcha del conocimiento en la disciplina e ignorar la pluralidad metodológica y la diversidad teórica producidas. El no ser capaz de seguir los avances en la disciplina y abusar de las técnicas (cuantitativa y cualitativa) para enmascarar su falta de lógica es equivalente a la falta de método y pensamiento propuestos por Sartori, lo que configura la ignorancia del pensador inconsciente. Permítanme explorar dos ejemplos para explicar cómo ocurre la ignorancia disciplinaria, por qué es problemática y cómo un pensador consciente puede corregirla.

Gary y Martínez (2016) ciertamente no entendían lo que un pensador consciente es. Los autores en una encuesta pobre y sesgada afirman que no hay producción teórica latinoamericana en RRII entre 2006 y 2014, lo cual puede ser fácilmente refutado con un vistazo rápido a los programas de la Conferencia Anual de la Asociación de Estudios Internacionales (ISA). De hecho, durante el periodo de tiempo elegido por los autores no hubo producción teórica en RRII en América Latina, como "brillantemente" enuncian. El problema es que no ha habido ningún tipo de producción teórica relevante en el mundo desde Alexander Wendt (1992; 1994; 1995; 1999). ¡Siendo precisamente por eso que ocurre el giro del sur global!

Al mismo tiempo que la disciplina discute cómo repensar las RRII y la expansión de diferentes puntos de vista sobre las diferentes realidades de estas, explora las limitaciones de las teorías producidas en el centro anglosajón de la disciplina. El tema especial del European Journal of International Relations en 2013 fue la quintaesencia de esta rama de la disciplina, arguyendo sobre la posibilidad de que la dis- 
ciplina hubiese alcanzado su fin en producción teórica (o al menos su extenuación). El ejercicio intelectual de producir o inclusive solo estimular la producción de conocimiento autóctono no solo hace referencia a la teorización, también intenta establecer una identidad disciplinaria y una metodología distintiva.

Por otro lado, cuando un académico es consciente sobre su disciplina, la identificación de un problema conceptual no proviene de montañas de datos. Es precisamente lo opuesto. El concepto de potencias medias en RRII es un buen ejemplo. A pesar de la gran cantidad de literatura sobre poderes medios, el concepto todavía es impreciso y usualmente confundido con mercados emergentes o paises intermedios.

Burges (2013), trabajando en la caracterización de Brasil como una potencia media, ilustra perfectamente lo que es un pensador consciente corrigiendo el problema de la malformación de conceptos, al tiempo que considera que "los académicos se centran en las características materiales de Brasil para sugerir que debe ser considerado como una potencia media, o estiran aproximaciones comportamentales al concepto para incluir características exhibidas por los posibles nuevos participantes como Brasil" (pp. 286-287)². Continúa, identificando por qué el estiramiento conceptual es problemático para su investigación

This aspect of connotative precision is central because the term 'middle power' is indelibly bound up with the foreign policy practices and traditions of countries such as Australia and Canada, not least because the concept was elaborated by diplomats in these countries as a foreign policy strategy and then further developed by academics focusing on these two cases.

Avoiding conceptual straining is also particularly important on the identity level of foreign policy. How a country identifies itself and sees itself in the global arena has an impact on the sort of foreign policy it constructs and pursues (Burges 2013, p. 287).

2 Traducción propia. 
Toneladas de datos sobre la política exterior de Brasil hubieran sido de poca utilidad si el autor no hubiera establecido desde el principio lo que él considera como una potencia media (Burges 2013, pp. 288-290), aceptando pasivamente el estiramiento conceptual para acomodar al país dentro de un concepto utilizado erróneamente. La respuesta de Burges es simple y clara: hemos confundido a Brasil como una potencia media.

\section{Observaciones finales}

Sartori (2004) es contundente en su conclusión cuando pregunta hacia dónde se dirige la ciencia política: "brevemente puesto, pensar antes de contar; y, también, use lógica al pensar". La disciplina y sus subcampos solo pueden avanzar si nos movemos hacia un medio más plural, en el que la ignorancia científica sea lentamente eliminada por la precisión conceptual. Durante al menos diez años, los politólogos han estado discutiendo si la democracia significa lo mismo que significó en las repercusiones que se tuvieron tras las guerras mundiales; mientras tanto las noticias falsas - fake news-, hechos alternativos —alternative facts_ y la manipulación de las elecciones demuestran lo contrario. Si continuamos fallando en confrontar la relación teoríapráctica, hemos creado una ciencia inútil. Sartori nunca ha sido tan actual y necesario.

\section{Referencias}

Armijo, L. (11 de noviembre de 2015). Entrevista realizada por el Fabricio Chagas Bastos.

Burges, S. W. (2013). Mistaking Brazil for a middle power. Journal of Iberian and Latin American Research, 19(02), 286-302.

Chagas-Bastos, F. H. (2017). 'From the periphery, with love': Recognition, status and agency in the Global South. Mimeo.

Garay, J., \& Martínez, M. (2016). Nada nuevo qué contar: la irrelevancia de los aportes a la teoría de las relaciones internacionales en América Latina. Oasis, 23, 31-52. 
Lima, L. (2011). Worlding Brazil: The theory of emotional action and the development of thinking about security in Brazil (1930 -2010), (Tesis doctoral, Aberystwyth, Aberystwyth University).

Sartori, G. (1970). Concept misformation in comparative politics. The American Political Science Review, 64(04), 1033-1053.

Sartori, G. (2004). Where is political science going? PS: Political Science \& Politics. 37(4), 785-787.

Wendt, A. (1992). Anarchy is what states make of it: The social construction of power politics. International Security, 46(02), 391-425.

Wendt, A. (1994). Collective identity formation and the international state. American Political Science Review, 88(02), 384-396.

Wendt, A. (1995). Constructing international politics. International Security, 20(01), 71-81.

Wendt, A. (1999). Social theory of internationalpolitics. Cambridge: Cambridge University Press. 